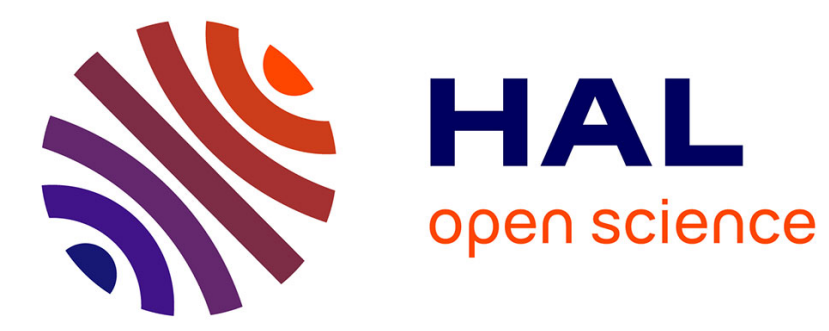

\title{
Discrete Walrasian Exchange Process
}

Jean-Marc Bonnisseau, Orntangar Nguenamadji

\section{To cite this version:}

Jean-Marc Bonnisseau, Orntangar Nguenamadji. Discrete Walrasian Exchange Process. 2009. halshs00442865

\section{HAL Id: halshs-00442865 \\ https://shs.hal.science/halshs-00442865}

Submitted on 23 Dec 2009

HAL is a multi-disciplinary open access archive for the deposit and dissemination of scientific research documents, whether they are published or not. The documents may come from teaching and research institutions in France or abroad, or from public or private research centers.
L'archive ouverte pluridisciplinaire HAL, est destinée au dépôt et à la diffusion de documents scientifiques de niveau recherche, publiés ou non, émanant des établissements d'enseignement et de recherche français ou étrangers, des laboratoires publics ou privés. 


\section{Documents de Travail du Centre d'Economie de la Sorbonne}

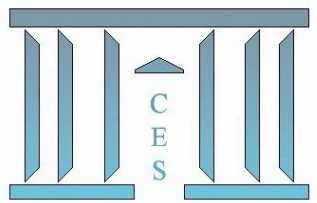

Discrete Walrasian Exchange Process

Jean-Marc BonNIssEAU, Orntangar NGUENAMADJI

2009.85

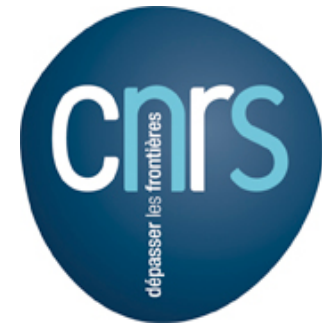




\title{
Discrete Walrasian Exchange Process*
}

\author{
Jean-Marc Bonnisseau and Orntangar Nguenamadji ${ }^{\dagger}$
}

December 2009

\begin{abstract}
In an exchange economy, we provide a discrete exchange process, which is Walrasian since the trades are given by the equilibrium allocation of the local equilibrium. We prove that this process attains a Pareto optimal allocation after a finite number of steps and the local equilibrium price then supports the Pareto optimal allocation. Furthermore, along the process, the allocation is feasible and the utility of each consumer is non-decreasing.
\end{abstract}

JEL classification: C62, D50, D62.

Keywords: Exchange process, Walrasian equilibrium, local equilibrium, Pareto optimum

\section{Introduction}

This paper takes place in the line of research on non-tâtonnement processes in exchange economies for which trading out of equilibrium is allowed. Chapter 13 of [1] provides a survey of the early works of the sixties, then [8], [9] and [5] have extended the study by weakening the assumptions or by proposing new rules for the price adjustment. But our contribution is mainly a continuation of [4] since the trade at each step is determined endogenously by a Walrasian equilibrium of an associated economy, which depends on the state of the process.

Nevertheless, our process exhibits a particular feature, which was never considered previously. Indeed, we consider a discrete process, which means that we have at most a countable number of steps and the trades at each step are not infinitesimal. Actually, we prove that the process reaches a Pareto

\footnotetext{
*We are grateful to Bernard Cornet for very valuable discussions on this paper and remarks on a previous version.

†Paris School of Economics and Université Paris 1 Panthéon-Sorbonne, Centre d'Economie de la Sorbonne-CNRS, 106-112 Boulevard de l'Hôpital, 75647 Paris Cedex 13, France. E-mail: Jean-Marc.Bonnisseau@univ-paris1.fr, Orntangar.Nguenamadji@univ-paris1.fr
} 
optimal feasible allocation after a finite number of steps. So, the convergence result is not an asymptotic one in the sense that we do not consider a limit after an infinite number of steps, but we prove that the process stops after having reach exactly an optimal allocation.

The process is built upon the following elementary mechanism. At a given step, taken the allocations of the agent as initial endowments, the exchanges are given by the allocations associated to a local equilibrium. Local equilibria are defined in a previous paper ([2]), where the main properties are given. Roughly speaking, a local equilibrium is a Walrasian equilibrium, where the consumers maximizes their preferences over a narrow set of possible trades instead of considering all possible trades. The key feature of a local equilibrium is the uniqueness in terms of price and allocation. So, the process is well defined.

The difference between our approach and the one of [4] comes from the fact that these authors considered a linear tangent economy to defined the infinitesimal trades. Actually, an equilibrium of the linear tangent economy is a limit of local equilibria when the consumers maximize over smaller and smaller sets of possible trades. Hence, our process can be interpreted as the discretization of the process of [4]. The advantage of this discretization comes from the uniqueness of local equilibria. So, we have a unique path, whereas a multi-valued differential equation is needed in the continuous time case.

Beyond the uniqueness, the local equilibrium enjoys also the nice property to be computable in the sense that it is a solution of a convex mathematical programming problem, for which efficient algorithms are known. So, the computation of the process is possible with efficient numerical technics.

To summarize, starting from an exchange economy with $\ell$ commodities and $m$ consumers where the initial endowments are denoted e, we get a finite sequence $\left.\left(p^{\nu},\left(x_{i}^{\nu}\right)\right)_{\nu=1}^{n}\right)$ such that: $\left(p^{\nu+1},\left(x_{i}^{\nu+1}\right)\right)$ is a local equilibrium of the economy with initial endowments $\left(x_{i}^{\nu}\right)$ with $\left(x_{i}^{0}\right)=\left(e_{i}\right)$; the allocation $\left(x_{i}^{\nu}\right)$ is feasible; the utility of each consumer is non-decreasing; the net trades $x_{i}^{\nu+1}-x_{i}^{\nu}$ have a zero value with respect to the current price $p^{\nu+1}$; and, at the end of the process, $\left(x_{i}^{n}\right)$ is a Pareto optimal allocation of the exchange economy and $p^{n}$ is supporting the allocation $\left(x_{i}^{n}\right)$.

With such non-tâtonnement process, we cannot expect to converge to a Walras equilibrium of the economy or to an element in the core. But, in [5], the authors are able to get such result for quasi-linear utility functions. This is a matter of further researches to check whether it works for our discrete process. In [3], it is shown that the Walrasian exchange process of [4] allows to converge to any individually rational Pareto optimal allocations with the right choice of the parameters, which determine the size of the infinitesimal trades. Since our process is a discretization of this continuous process, we can expect to get the same neutrality property but this remains to be done. 


\section{A discrete walrasian process}

We consider a pure exchange economy with $\ell$ commodities and $m$ consumers ${ }^{1}$. The consumption sets are $\mathbb{R}_{+}^{\ell}$, the preferences of the consumers are represented by a utility function $u_{i}$ from $\mathbb{R}_{+}^{\ell}$ to $\mathbb{R}$ and the initial vector of endowments is denoted $\underline{\mathbf{e}}$. That is, $\mathcal{E}(\underline{\mathbf{e}})=\left(\mathbb{R}_{+}^{\ell}, u_{i}, \underline{e}_{i}\right)_{i=1}^{m}$. We posit the following classical assumptions:

Assumption C. For all $i$,

a) (Differentiable monotonicity) $u_{i}$ is twice continuously differentiable on $\mathbb{R}_{++}^{\ell}$ and $\nabla u_{i}\left(x_{i}\right) \in \mathbb{R}_{++}^{\ell}$ for all $x_{i} \in \mathbb{R}_{++}^{\ell} ;$

b) (Differentiable strict quasi-concavity) for all $x_{i} \in \mathbb{R}_{++}^{\ell}$ and for all $z_{i} \in$ $\mathbb{R}^{\ell} \backslash\{0\}$, one has $\left[\nabla u_{i}\left(x_{i}\right) \cdot z_{i}=0\right] \Rightarrow\left[z_{i} \cdot D^{2} u_{i}\left(x_{i}\right)\left(z_{i}\right)<0\right]$;

c) (Boundary behavior) for all $\underline{x}_{i} \in \mathbb{R}_{++}^{\ell}$, the set $U_{i}\left(\underline{x}_{i}\right)=\left\{x_{i} \in \mathbb{R}_{++}^{\ell} \mid u_{i}\left(x_{i}\right) \geq u_{i}\left(\underline{x}_{i}\right)\right\}$ is a closed subset of $\mathbb{R}^{\ell} ;$

d) (Survival condition) $\underline{e}_{i} \in \mathbb{R}_{++}^{\ell}$.

Let us recall the definition of a $\tau$-local equilibrium introduced in [2].

Definition 2.1 For $\tau \in\left([0,1]^{\ell}\right)^{m}$, a $\tau$-local equilibrium of $\mathcal{E}(\underline{\mathbf{e}})$ is a Walras equilibrium (with a normalized price in the simplex) of the $\tau$-local economy $\mathcal{E}^{\tau}(\underline{\mathbf{e}})=\left(\left\{\left(\mathbf{1}-\tau_{i}\right) \underline{e}_{i}\right\}+\mathbb{R}_{+}^{\ell}, u_{i}, \underline{e}_{i}\right)_{i=1}^{m}$.

Let $\mathbf{E}:=\left\{\left(x_{i}\right) \in\left(\mathbb{R}_{++}^{\ell}\right)^{m} \mid \sum_{i=1}^{m}\left(x_{i}-\underline{e}_{i}\right)=0, u_{i}\left(x_{i}\right) \geq u_{i}\left(\underline{e}_{i}\right) \forall i\right\}$. From Assumption C(c), E is a closed subset of $\left(\mathbb{R}^{\ell}\right)^{m}$ and it is clearly bounded, so it is a compact subset of $\left(\mathbb{R}^{\ell}\right)^{m}$. We now recall the main properties of local equilibria proved in [2].

Proposition 2.1 Let $\Gamma \subset \mathbb{R}_{++}^{\ell} \cup\{\mathbf{0}\}$ be a nonempty closed convex cone. Under Assumption $C$, there exists $\left.\bar{\theta} \in] 0,1[\text { such that, for all } \tau \in(\Gamma \cap] 0, \bar{\theta}]^{\ell}\right)^{m}$ and for all $\mathbf{e} \in \mathbf{E}$, the economy $\mathcal{E}(\mathbf{e})$ has a unique normalized $\tau$-local equilibrium denoted $W(\mathbf{e}, \tau)$. Furthermore, $W$ is a continuous mapping from $\left.\mathbf{E} \times(\Gamma \cap] 0, \bar{\theta}]^{\ell}\right)^{m}$ to $S \times\left(\mathbb{R}_{+}^{\ell}\right)^{m}$.

\subsection{The discrete Walrasian exchange process}

In the following, we consider a given closed convex cone $\Gamma \subset \mathbb{R}_{++}^{\ell} \cup\{\mathbf{0}\}$ and a positive lower bound $\underline{\theta}<\bar{\theta}$ where $\bar{\theta}$ is given by proposition 2.1. Then, in the remaining of the paper, the parameters $\tau$ are always taken in $\Theta=$ $\left(\Gamma \cap[\underline{\theta}, \bar{\theta}]^{\ell}\right)^{m}$.

\footnotetext{
${ }^{1}$ In $\mathbb{R}^{\ell}, S$ denotes the simplex and $\|x\|=\sum_{h=1}^{\ell}\left|x_{h}\right|$ for all $x \in \mathbb{R}^{\ell}$. The box product is defined as follows: for a pair of vectors $(x, y)$ of $\mathbb{R}^{\ell}, x \square y$ is the vector of $\mathbb{R}^{\ell}$ with components $x^{h} y^{h}$ for $h=1, \ldots, \ell$. We denote by $\mathbf{1}$ the vector of $\mathbb{R}^{\ell}$ the components of which are all equal to 1 .
} 
A discrete Walrasian exchange process controlled by a sequence $(\tau(t))_{t \in \mathbb{N}}$ of $\Theta$ is a sequence $(p(t), x(t))_{t \in \mathbb{N}^{*}}$ in $S \times\left(\mathbb{R}_{+}^{\ell}\right)^{m}$ defined by the following discrete dynamical system:

$$
(p(t+1), x(t+1))=W(x(t), \tau(t)), \quad x(0)=\underline{\mathbf{e}}
$$

In other words, a discrete Walrasian exchange process starting from $\underline{\mathbf{e}}$ and controlled by the sequence $(\tau(t))_{t \in \mathbb{N}}$ is a sequence $(x(t), p(t))_{t \in \mathbb{N}^{*}}$ in $\mathbf{E} \times S$ such that for all $t \in \mathbb{N},(x(t+1), p(t+1))$ is the unique $\tau(t)$-local equilibrium of $\mathcal{E}(x(t))$.

In the following proposition, we gather the first properties of a discrete Walrasian exchange process.

Proposition 2.2 The discrete Walrasian exchange process $(p(t), x(t))_{t \in \mathbb{N}^{*}}$ starting from e satisfies the following conditions:

1. $u_{i}\left(x_{i}(t+1)\right) \geq u_{i}\left(x_{i}(t)\right) \geq u_{i}\left(\underline{e}_{i}\right)$ for all $i$ and for all $t$.

2. $p(t+1) \cdot\left(x_{i}(t+1)-x_{i}(t)\right)=0$ for all $i$ and all $t$.

3. $\sum_{i=1}^{m} x_{i}(t)=\sum_{i=1}^{m} \underline{e}_{i}$ for all $t \in \mathbb{N}$.

4. $x(t+1)=x(t)$ if and only if $(x(t))$ is a Pareto optimum for $\mathcal{E}(\underline{\mathbf{e}})$. Furthermore, in that case, $p(t)$ is a price supporting $x(t)$ that is, $p(t)$ is positively proportional to $\nabla u_{i}\left(x_{i}(t)\right)$ for all $i$.

The above proposition means that the utility level of each agent is nondecreasing, the allocation is individually rational and feasible. The exchange are done according to the rate of exchange given by the price $p(t)$ at each step. Finally, the equilibrium point of the process, that is the point satisfying $x(t+1)=x(t)$ are included in the set of individually rational Pareto Optimal allocations.

Proof. The first three statements are consequences of the well-known properties of a Walras equilibrium, namely, the allocations are individually rational, the Walras law and the market clearing condition.

As for Property (4), if $(x(t)$ is a Pareto optimum, then $(p, x(t))$, where $p=$ $\left(1 /\left(\sum_{h=1}^{\ell} \frac{\partial u_{1}}{\partial x_{h}}\left(x_{1}(t)\right)\right)\right) \nabla u_{1}\left(x_{1}(t)\right)$, is the unique equilibrium of the economy $\mathcal{E}(x(t))=\left(\mathbb{R}_{+}^{\ell}, u_{i}, x_{i}(t)\right)_{i=1}^{m}$. Then, it is the unique $\tau$-local equilibrium of the economy for any $\tau \in \Theta$. Consequently, $x(t+1)=x(t)$.

If $x(t+1)=x(t)$, then for all $i, x_{i}(t+1) \gg\left(\mathbf{1}-\tau_{i}(t)\right) x_{i}(t)$. Hence, the first order necessary condition of optimality at the demand $x_{i}(t+1)$ implies that $\nabla u_{i}\left(x_{i}(t+1)\right)$ is positively proportional to $p(t+1)$. Consequently, $x(t+1)$ is a feasible allocation of the economy $\mathcal{E}(\underline{\mathbf{e}})$ and the gradient vectors of the utility functions are positively proportional to the same vector $p(t+1)$, which characterizes a Pareto optimal allocations. 


\section{$2.2 \quad$ Statement of the result}

The following proposition presents our main result.

Proposition 2.3 Let $(\tau(t))_{t \in \mathbb{N}}$ be a sequence of $\Theta$ and $(p(t), x(t))_{t \in \mathbb{N}}$ a discrete Walrasian exchange process controlled by $(\tau(t))_{t \in \mathbb{N}}$ associated to the pure exchange economy $\mathcal{E}(\underline{\mathbf{e}})$. Then, there exists $t_{0} \in \mathbb{N}$ such that for all $t \geq t_{0}$, one has:

$$
(p(t+1), x(t+1))=(p(t), x(t)) .
$$

In words, the discrete Walrasian exchange process attains an equilibrium point after a finite number of steps. From Proposition 2.2, the equilibrium point is an individually rational Pareto optimum. Furthermore, the equilibrium price $p\left(t_{0}\right)$ is a supporting price of the optimal allocation $x\left(t_{0}\right)$. As each step is given by a unique and calculable Walrasian equilibrium, the process is totally calculable.

So contrary to the usual continuous-time process, the Pareto optimal allocation is not the limit when time tends to $+\infty$ but it is obtain exactly after a finite number of iterations.

The proof of this proposition is the purpose of the next section. The main steps of the proof are the following: a unique step is sufficient to reach a Pareto optimal allocation if the initial endowments lie in some neighborhood of the set of individually rational Pareto optima and the aggregated utility gain is bounded below by a positive lower bound on the complementary of this neighborhood in $\mathbf{E}$.

\section{Proof of Propositions 2.3}

For each $i$ let us define $\hat{\mathbf{E}}_{i}=\left\{x_{i} \in \mathbb{R}_{++}^{\ell} \mid \exists x_{-i} \in\left(\mathbb{R}_{++}\right)^{m-1},\left(x_{i}, x_{-i}\right) \in \mathbf{E}\right\}$. Then $\hat{\mathbf{E}}_{i}$ is a compact subset of $\mathbb{R}_{++}^{\ell}$. From [?] Proposition 2.6.4, under Assumptions $\mathrm{C}$, the preferences of agent $i$ can be represented by a utility function satisfying Assumption $\mathrm{C}$, which is furthermore strictly concave on a convex neighborhood of $\hat{\mathbf{E}}_{i}$. Consequently, we assume in the following that the utility functions $u_{i}$ are strictly concave on a convex neighborhood of $\hat{\mathbf{E}}_{i}$.

In the following, we denote by $\mathcal{P}_{I R}$ the set of individually rational Pareto Optima of $\mathcal{E}(\underline{\mathbf{e}})$, that is the Pareto optimal allocation $\left(x_{i}\right)$ satisfying $u_{i}\left(x_{i}\right) \geq$ $u_{i}\left(\underline{e}_{i}\right)$ for all $i$. For $(\mathbf{e}, \tau) \in \mathbf{E} \times \Theta, W_{0}(\mathbf{e}, \tau)$ denotes the $\tau$-local equilibrium price vector and $W_{i}(\mathbf{e}, \tau)$ denotes the $\tau$-local equilibrium allocation of the $i$ th consumer.

Lemma 3.1 There exists $\varepsilon>0$ such that for all $(\mathbf{e}, \tau) \in B\left(\mathcal{P}_{I R}, \varepsilon\right) \times \Theta$, one has $\left(W_{i}(\mathbf{e}, \tau)\right)_{i=1}^{m} \in \mathcal{P}_{I R}$.

The proof is given in Appendix. In the following, we denote by $\overline{\mathbf{E}}$ the set $\mathbf{E} \backslash B\left(\mathcal{P}_{I R}, \varepsilon\right)$, where $\varepsilon$ is given by Lemma 3.1. The next step is to prove the following lemma. 
Lemma 3.2 There exists $\underline{\delta}>0$ such that for all $\mathbf{e} \in \overline{\mathbf{E}}$ and for all $\tau \in \Theta$ one has:

$$
\left.\sum_{i=1}^{m}\left[u_{i}\left(W_{i}(\mathbf{e}, \tau)\right)-u_{i}\left(e_{i}\right)\right)\right] \geq \underline{\delta} .
$$

We prepare the proof by some preliminary results. Let $(\mathbf{e}, \tau) \in \mathbf{E} \times \Theta$. Let $\left(p^{*}, x^{*}\right)$ be the $\tau$-local equilibrium of $\mathcal{E}(\mathbf{e})$. Since the utility functions are strictly increasing, the budget constraints are binding at equilibrium. Note also that the Kuhn-Tucker first order conditions for the consumer optimization problem are sufficient since $u_{i}$ is concave. Formally, for all $i$, there exists $\lambda_{i}^{*}(\mathbf{e}, \tau) \geq 0$ and $\mu_{i}^{*}(\mathbf{e}, \tau) \geq 0$ such that

$$
\begin{array}{r}
\nabla u_{i}\left(x_{i}^{*}\right)=\lambda_{i}^{*}(\mathbf{e}, \tau) p^{*}-\mu_{i}^{*}(\mathbf{e}, \tau), \\
\mu_{i}^{*}(\mathbf{e}, \tau) \cdot\left(-x_{i}^{*}+\left(\mathbf{1}-\tau_{i}\right) \square e_{i}\right)=0, x_{i}^{*} \geq\left(\mathbf{1}-\tau_{i}\right) \square e_{i} \\
p^{*} \cdot x_{i}^{*}=p^{*} \cdot e_{i} .
\end{array}
$$

Note that $\lambda_{i}^{*}(\mathbf{e}, \tau)>0$ since $x_{i}^{*}-\left(\mathbf{1}-\tau_{i}\right) \square e_{i} \in \mathbb{R}_{+}^{\ell} \backslash\{0\}, \nabla u_{i}\left(x_{i}^{*}\right) \gg 0$ and

$$
\lambda_{i}^{*}(\mathbf{e}, \tau)=\frac{\nabla u_{i}\left(x_{i}^{*}\right) \cdot\left(x_{i}^{*}-\left(\mathbf{1}-\tau_{i}\right) \square e_{i}\right)}{p^{*} \cdot\left(\tau_{i} \square e_{i}\right)}
$$

Now, we can consider the following optimization problems on which the proof of Lemma 3.2 is built.

$$
\begin{gathered}
\mathcal{Q}_{e q}(\mathbf{e}, \tau):\left\{\begin{array}{l}
\operatorname{maximize} \sum_{i=1}^{m} \frac{1}{\lambda_{i}^{*}(\mathbf{e}, \tau)} u_{i}\left(x_{i}\right) \\
\sum_{i=1}^{m}\left(x_{i}-e_{i}\right)=0 \\
x_{i} \geq\left(\mathbf{1}-\tau_{i}\right) \square e_{i}
\end{array}\right. \\
\mathcal{Q}_{o p}(\mathbf{e}, \tau):\left\{\begin{array}{l}
\operatorname{maximize} \sum_{i=1}^{m} \frac{1}{\lambda_{i}^{*}(\mathbf{e}, \tau)} u_{i}\left(x_{i}\right) \\
\sum_{i=1}^{m}\left(x_{i}-e_{i}\right)=0 \\
u_{i}\left(x_{i}\right) u_{i}\left(e_{i}\right), i=1, \ldots, m \\
x_{i} \geq 0 i=1, \ldots, m
\end{array}\right.
\end{gathered}
$$

Remark 3.1 a) $x^{*}$ is a solution of $\mathcal{Q}_{e q}(\mathbf{e}, \tau)$. Actually, from Condition (2), we have the following conditions which are sufficient for $\mathcal{Q}_{e q}(\mathbf{e}, \tau)$ thanks to the concavity of $u_{i}$ : for all $i$,

$$
\left\{\begin{array}{l}
\frac{1}{\lambda_{i}^{*}(\mathbf{e}, \tau)} \nabla u_{i}\left(x_{i}^{*}\right)=p^{*}-\frac{1}{\lambda_{i}^{*}(\mathbf{e}, \tau)} \mu_{i}^{*}(\mathbf{e}, \tau) \\
\mu_{i}^{*}(\mathbf{e}, \tau) \cdot\left(-x_{i}^{*}+\left(\mathbf{1}-\tau_{i}\right) \square e_{i}\right)=0
\end{array}\right.
$$

b) A solution $\bar{x}$ of $\mathcal{Q}_{o p}(\mathbf{e}, \tau)$ is an element of $\mathcal{P}_{I R}$ since $\mathbf{e} \in \mathbf{E}$, that is, $u_{i}\left(\bar{x}_{i}\right) \geq u_{i}\left(e_{i}\right) \geq u_{i}\left(\underline{e}_{i}\right)$ for all $i$.

Lemma 3.3 (i) For all $(\mathbf{e}, \tau) \in \overline{\mathbf{E}} \times \Theta$ and for all solution $\bar{x}$ of $\mathcal{Q}_{o p}(\mathbf{e}, \tau)$, $u_{i}\left(\bar{x}_{i}\right) \geq u_{i}\left(e_{i}\right)$ for all $i$ and there exists $i_{0}$, such that $u_{i_{0}}\left(\bar{x}_{i_{0}}\right)>u_{i_{0}}\left(e_{i_{0}}\right)$.

(ii) There exists $\rho>0$ such that $\sum_{i=1}^{m} \frac{1}{\lambda_{i}^{*}(\mathbf{e}, \tau)}\left[u_{i}\left(\bar{x}_{i}\right)-u_{i}\left(e_{i}\right)\right] \geq \rho$ for all $(\mathbf{e}, \tau) \in \overline{\mathbf{E}} \times \Theta$ and for all solution $\bar{x}$ of $\mathcal{Q}_{o p}(\mathbf{e}, \tau)$. 
Proof. See Appendix.

Lemma 3.4 For all $(\mathbf{e}, \tau) \in \overline{\mathbf{E}} \times \Theta$,

$$
\sum_{i=1}^{m} \frac{1}{\lambda_{i}^{*}(\mathbf{e}, \tau)}\left[u_{i}\left(x_{i}^{*}\right)-u_{i}\left(e_{i}\right)\right] \geq \underline{\theta} \sum_{i=1}^{m} \frac{1}{\lambda_{i}^{*}(\mathbf{e}, \tau)}\left[u_{i}\left(\bar{x}_{i}\right)-u_{i}\left(e_{i}\right)\right]
$$

where $x^{*}$ is the solution of $\mathcal{Q}_{e q}(\mathbf{e}, \tau)$ and $\bar{x}$ is the solution of $\mathcal{Q}_{o p}(\mathbf{e}, \tau)$.

Proof of Lemma 3.4 . Let $\xi_{i}=(1-\underline{\theta}) e_{i}+\underline{\theta} \bar{x}_{i}$ for all $i$. Then, since $\tau_{i}^{h} \geq \underline{\theta}$ for all $(i, h)$, one has $\xi_{i} \geq\left(\mathbf{1}-\tau_{i}\right) \square e_{i}$ for all $i$ and $\sum_{i=1}^{m} \xi_{i}=\sum_{i=1}^{m} e_{i}$. So, since $x^{*}$ is a solution of $\mathcal{Q}_{e q}(\mathbf{e}, \tau)$ :

$$
\sum_{i=1}^{m} \frac{1}{\lambda_{i}^{*}(\mathbf{e}, \tau)}\left[u_{i}\left(x_{i}^{*}\right)-u_{i}\left(\xi_{i}\right)\right] \geq 0
$$

Furthermore, since $u_{i}$ is concave for all $i$,

$$
\sum_{i=1}^{m} \frac{1}{\lambda_{i}^{*}(\mathbf{e}, \tau)}\left[u_{i}\left(x_{i}^{*}\right)-(1-\underline{\theta}) u_{i}\left(e_{i}\right)-\underline{\theta} u_{i}\left(\bar{x}_{i}\right)\right] \geq \sum_{i=1}^{m} \frac{1}{\lambda_{i}^{*}(\mathbf{e})}\left[u_{i}\left(x_{i}^{*}\right)-u_{i}\left(\xi_{i}\right)\right]
$$

Consequently $\sum_{i=1}^{m} \frac{1}{\lambda_{i}^{*}(\mathbf{e}, \tau)}\left[u_{i}\left(x_{i}^{*}\right)-(1-\underline{\theta}) u_{i}\left(e_{i}\right)-\underline{\theta} u_{i}\left(\bar{x}_{i}\right)\right] \geq 0$ that is:

$$
\sum_{i=1}^{m} \frac{1}{\lambda_{i}^{*}(\mathbf{e}, \tau)}\left[u_{i}\left(x_{i}^{*}\right)-u_{i}\left(e_{i}\right)\right] \geq \underline{\theta} \sum_{i=1}^{m} \frac{1}{\lambda_{i}^{*}(\mathbf{e}, \tau)}\left[u_{i}\left(\bar{x}_{i}\right)-u_{i}\left(e_{i}\right)\right]
$$

Remark 3.2 Since $\mathbf{E} \times \Theta$ is compact and $\lambda_{i}^{*}(\cdot, \cdot)$ is continuous and positive on $\mathbf{E} \times \Theta$ for all $i$, there exists $\underline{\lambda}>0$ such that for all $(\mathbf{e}, \tau) \in \mathbf{E} \times \Theta$ and for each $i$, one has:

$$
\lambda_{i}^{*}(\mathbf{e}, \tau) \geq \underline{\lambda} .
$$

\section{Proof of Lemma 3.2 .}

From Lemmata 3.3 and 3.4, for all $\mathbf{e} \in \overline{\mathbf{E}}$ and for all $\tau \in \Theta$, we have:

$$
\sum_{i=1}^{m} \frac{1}{\lambda_{i}^{*}(\mathbf{e}, \tau)}\left[u_{i}\left(x_{i}^{*}\right)-u_{i}\left(e_{i}\right)\right] \geq \underline{\theta} \rho>0
$$

Since, from Remark $3.2, \lambda_{i}^{*}(\mathbf{e}, \tau) \geq \underline{\lambda}$, we get finally:

$$
\sum_{i=1}^{m}\left[u_{i}\left(x_{i}^{*}\right)-u_{i}\left(e_{i}\right)\right] \geq \underline{\lambda} \underline{\theta} \rho .
$$

We get then the result by taking $\underline{\delta}=\underline{\lambda} \underline{\theta} \rho$ 
Proof of Proposition 2.3. Note that if there exists $\hat{t}$ such that $x(\hat{t}) \in$ $B\left(\mathcal{P}_{I R}, \varepsilon\right)$ then the result holds from Lemma 3.1 with $t_{0}=\hat{t}+1$.

Assume that for all $t, x(t) \notin B\left(\mathcal{P}_{I R}, \varepsilon\right)$. Then from Lemma 3.2, we deduce the following:

$$
\sum_{i=1}^{m}\left[u_{i}\left(x_{i}(t+1)\right)\right] \geq t \underline{\delta}+\sum_{i=1}^{m} u_{i}\left(\underline{e}_{i}\right)
$$

That is, $\sum_{i=1}^{m}\left[u_{i}\left(x_{i}(t+1)\right)\right] \rightarrow+\infty$ when $t \rightarrow+\infty$. But this is not possible since $x(t) \in \mathbf{E}$ for all $t \in \mathbb{N}$ and $\mathbf{E}$ is compact.

\section{Appendix}

Proof of Lemma 3.1 Using the same argument as in the last part of the proof of Proposition 2.2, it suffices to show that there exists $\varepsilon>0$ such that for all $\left.(\mathbf{e}, \tau) \in B\left(\mathcal{P}_{I R}, \varepsilon\right) \times \Theta\right), W_{i}(\mathbf{e}, \tau) \gg\left(\mathbf{1}-\tau_{i}\right) e_{i}$ for all $i$. Assume that it is false. That is, for all $\nu \in \mathbb{N}^{*}$ there exists $\mathbf{e}^{\nu} \in \mathbf{E}, \tau^{\nu} \in \Theta$ and $\left(i_{0}^{\nu}, h_{0}^{\nu}\right) \in\{1, \ldots, m\} \times\{1, \ldots, \ell\}$ such that

$$
\mathbf{e}^{\nu} \in B\left(\mathcal{P}_{I R},(1 / \nu)\right) \text { and } W_{i_{0}^{\nu} h_{0}^{\nu}}\left(\mathbf{e}^{\nu}, \tau^{\nu}\right)=\left(1-\tau_{i_{0}^{\nu}}^{\nu} h_{0}^{\nu}\right) e_{i_{0}^{\nu} h_{0}^{\nu}}^{\nu}
$$

Let $\left(\left(\mathbf{e}^{\nu}\right),\left(\tau^{\nu}\right),\left(i_{0}^{\nu}, h_{0}^{\nu}\right)\right)$ be the sequence of $\mathbf{E} \times \Theta \times(\{1, \ldots, m\} \times\{1, \ldots, \ell\})$ obtained by this way. As $\mathbf{E} \times \Theta$ is compact and $\{1, \ldots, m\} \times\{1, \ldots, \ell\}$ a finite set, we can find a convergent subsequence of $\left(\mathbf{e}^{\nu}, \tau^{\nu}\right)$, still denoted by $\left(\mathbf{e}^{\nu}, \tau^{\nu}\right)$ for the sake of simplicity, with a constant corresponding subsequence of $\left(i_{0}^{\nu}, h_{0}^{\nu}\right)$ denoted by $\left(i_{0}, h_{0}\right)$.

Since $\mathbf{e}^{\nu} \in B\left(\mathcal{P}_{I R}, \frac{1}{\nu}\right)$ and $W_{i_{0} h_{0}}\left(\mathbf{e}^{\nu}, \tau^{\nu}\right)=\left(1-\tau_{i_{0} h_{0}}^{\nu}\right) e_{i_{0} h_{0}}^{\nu}$ for all $\nu$, the limit $\overline{\mathbf{e}}$ belongs to $\mathcal{P}_{I R}$ and by the continuity of $W_{i_{0} h_{0}}$, one gets:

$$
W_{i_{0} h_{0}}(\overline{\mathbf{e}}, \bar{\tau})=\left(1-\bar{\tau}_{i_{0} h_{0}}\right) \bar{e}_{i_{0} h_{0}}
$$

where $\bar{\tau}$ is the limit of $\tau^{\nu}$. But we know also that $W_{i_{0} h_{0}}(\overline{\mathbf{e}}, \bar{\tau})=\bar{e}_{i_{0} h_{0}}$ since $W_{i}(\mathbf{e}, \tau)=e_{i}$ for all $i$ and for all $\mathbf{e} \in \mathcal{P}_{I R}$. We get then a contradiction since $\bar{\tau}_{i_{0} h_{0}} \geq \underline{\theta}>0$.

Proof of Lemma 3.3. Let $(\mathbf{e}, \tau) \in \overline{\mathbf{E}} \times \Theta$ and

$$
\bar{L}(\mathbf{e}, \tau)=\sum_{i=1}^{m} \frac{1}{\lambda_{i}^{*}(\mathbf{e}, \tau)}\left[u_{i}\left(\bar{x}_{i}\right)-u_{i}\left(e_{i}\right)\right]
$$

where $\bar{x}$ is a solution of $\mathcal{Q}_{o p}(\mathbf{e}, \tau)$.

(i) Assume that $u_{i}\left(\bar{x}_{i}\right)=u_{i}\left(e_{i}\right)$ for all $i$. Then $\mathbf{e}$ is also a solution of $\mathcal{Q}_{o p}(\mathbf{e}, \tau)$ and from Remark 3.1, it belongs to $\mathcal{P}_{I R}$ but this is a contradiction since $\mathbf{e} \in \overline{\mathbf{E}}$ and $\overline{\mathbf{E}} \cap \mathcal{P}_{I R}=\emptyset$. 
(ii) Note that from $(i)$ we have: $\bar{L}(\mathbf{e}, \tau)>0$ for all $(\mathbf{e}, \tau) \in \overline{\mathbf{E}} \times \Theta$. Recall that the $\tau$-local equilibrium mapping $W$ is continuous. So, $(\mathbf{e}, \tau) \mapsto \lambda_{i}^{*}(\mathbf{e}, \tau)$ is a continuous function for all $i$ since, as already noticed, the first order condition of optimality for the consumer's optimization problem leads to:

$$
\lambda_{i}^{*}(\mathbf{e}, \tau)=\frac{\nabla u_{i}\left(W_{i}(\mathbf{e}, \tau)\right) \cdot\left(W_{i}(\mathbf{e}, \tau)-\left(\mathbf{1}-\tau_{i}\right) \square e_{i}\right)}{\tau_{i} W_{0}(\mathbf{e}, \tau) \cdot e_{i}} .
$$

Hence $\bar{L}(\cdot, \cdot)$ is continuous and we get the result by taking

$$
\rho=\min _{(\mathbf{e}, \tau) \in \overline{\mathbf{E}} \times \Theta} \bar{L}(\mathbf{e}, \tau)
$$

which is positive since $\overline{\mathbf{E}} \times \Theta$ is compact and $\bar{L}(\mathbf{e}, \tau)>0$ for all $(\mathbf{e}, \tau) \in$ $\overline{\mathbf{E}} \times \Theta$.

\section{References}

[1] ARROW, K. and HANHN, F.H., General Competitive Analysis, San Francisco: Holden-Day, 1971.

[2] Bonnisseau, J-M and Nguenamadji, O., On the uniqueness of local equilibria, CES Working Papers, 2008.102.

[3] Bottazzi, J-M. Accessibility of Pareto optima by Walrasian Exchange processes. Journal of Mathematical Economics, Elsevier, vol. 23(6), pp. 585-603, 1994.

[4] Champsaur, P. and Cornet, B.: Walrasian Exchange Processes, in Economic Decision Making: Games, Economics and Optimisation, Contributions in Honor of Jacques H. Drèze, J.J. Gabszewicz et al (eds.), North Holland, Amsterdam, pp. 3-13, 1990.

[5] Chander, P. and Tulkens, H.:Exchange Processes, the Core and Competitive allocations, in Economic Decision Making: Games, Economics and Optimisation, Contributions in Honor of Jacques H. Drèze, J.J. Gabszewicz et al (eds.), North Holland, Amsterdam, pp. 15-32, 1990.

[6] Debreu, G., Economies with a Finite Set of Equilibria, Econometrica, Vol. 38, pp. 387-392, 1970.

[7] Dierker, E., Regular Economies , Handbook of Mathematical Economics, Vol. 1, 1981, PP. 795-830.

[8] Smale, S., "Exchange processes with price adjustment", Journal of Mathematical Economics, 3, 211-216, 1976.

[9] Tulkens, H. and Zamir, S., "Surplus-sharing Local Games in Dynamic Exchange Processes", The Review of Economic Studies, 46, 305-313, 1979 